\title{
Pattern of chronic myelogenous leukemia in the Congo
}

\author{
Lydie Ocini Ngolet $^{1} *$, Malanda Felix ${ }^{2}$, Alexis Elira Dokekias ${ }^{1}$
}

\begin{abstract}
Objective: Chronic myeloid leukemia is a clonal myelo proliférative disorder caused by reciprocal translocation $\mathrm{t}(9 ; 22)$ that induces tyrosin kinase protein. Imatinib is a selective inhibitor of this protein. Aim of this study is to describe, the clinical and biological feature of chronic myeloid leukemia in the Congo.

Material and Methods: The study is a retrospective study which conducted in Clinical Hematology unit of teaching hospital in Brazzaville, Congo on newly diagnosed patients with chronic myeloid leukemia. A total of 25 males and 14 females with a mean age of 36 years (16 and 69 years) at time of the diagnosis were enrolled in the study.

Results: The mean duration of the illness at the time of the diagnosis was 11.4 months (0.5 and 50 months). Patients presented with a splenomegaly, mean leukocytosis rate at $168.5 \mathrm{~g} / 1$ (14.6-381 g/l), moderate anemia at $8.4 \mathrm{~g} / \mathrm{dL}(3.1$ and $12.8 \mathrm{~g} / \mathrm{dl})$ and normal platelet rate at $415.4 \mathrm{~g} / \mathrm{dl}(164$ and $998 \mathrm{~g} / \mathrm{l})$. Thirty eight on thirty nine patients $(97.43 \%)$ presented at chronic phase and the Sokal score was high in $46.2 \%$. Imatinib induced complete hematologic response at 3 months in all patients. Complete cytogenetic response was achieved in $20.51 \%$. The median follow up period was months. The Overall Survival was $86.6 \%$ at 26 months and Progression Free Survival was $91.8 \%$ at 12 months.

Conclusion: This meta analysis report represents the clinical and biological pattern of chronic myeloid leukemia in the Congo.
\end{abstract}

Key words: chronic myeloid leukemia, pattern, Congo

\section{Introduction}

Chronic myelogenous leukemia (CML) is a malignant clonal alteration of the pluripotent hematopoietic stem cell. It is a triphasic disease with a chronic phase (CP) that lasts three to six years, followed by transformation to an accelerated and then a blastic phase, of short durations (1). Biologic basis has been elucidated. The characteristic abnormality of the CML is the Philadelphia chromosome $(\mathrm{Ph}+)$ that results from a reciprocal chromosomal translocation between chromosome 9 and chromosome 22 (2).The genetic consequence of this translocation is a genetic sequence fusion that forms the BCR-ABL oncogene, which encodes for a constitutively active Bcr-ABL kinase that mediates cellular transformation (3). CML has an annual worldwide incidence of $1 / 100.000$ with a male:female ration of $1.5: 1$ (4). CML is in the Congo the most frequent chronic leukemia with an incidence of 2.2/ 100.000 (5). The advent of imatinib at no cost in the Congo through North-south partnership has decreased the mortality rate related to CML and increased patients' overall survival (6). The aim of this report is to describe the clinical and biological pattern of CML in the Congo.

\section{Patients and Methods}

The descriptive retrospective study took place in the Hematology Unit at the teaching hospital, the largest hospital in the country, from January 2008 to December 2015 (8 years). The diagnosis of CML was established by using cytogenetic studies. Cytogenetic studies were performed in the Cytogenetic Laboratory in Kremlin Bicêtre Teaching Hospital (France). The technique performed was chromosome banding analysis of at least 20 bone marrow cell metaphases, after short-term and long-term culture with standard $G$ or $\mathrm{G}$ banding techniques.

\section{Inclusion criteria:}

- Newly diagnosed Phi + CML

- Complete medical record

\section{Non inclusion criteria:}

- $\quad$ CML not confirmed by cytogenetic analysis

- Incomplete medical record

\section{Cytogenetic and hematologic responses:}

Patients were enrolled in the Glivec Initiative Program Assistant Program (GIPAP) and were granted oral IM at the dosage of $400 \mathrm{mg}$ daily. The hematologic and 
cytogenetic responses to IM were analyzed with regard to the European Leukemia Net Guidelines and U.S National Comprehensive Cancer Network (NCCN) Guidelines.

Hematological response (HR) indicates improvement in peripheral blood cell counts. It may be complete [ CHR (White blood cell count below $10 \times 10^{9} / 1$, platelets below $450 \times 10^{9} / 1$, immature cells absent or normalized differential, no signs or symptoms of disease )] or partial ( persistence of immature cells , platelets below $50 \%$ of pre-treatment levels but above $\left(450 \times 10^{9} / 1\right)$.

A Cytogenetic Response (CYR) defines the proportion of $\mathrm{Ph}+$ cells identified in bone marrow or peripheral blood. CYR may be complete (CCYR): complete absence of $\mathrm{Ph}+$ cells, partial (PCYR) $1-35 \% \mathrm{Ph}$ cells, minor $(36-65 \% \mathrm{Ph}+$ cells $)$, or minimal $66-95 \% \mathrm{Ph}$ cells. A Major CYR (MCYR) is defined as CCRYR or PCYR. Loss of CYR is considered when an increase in $\mathrm{Ph}+$ metaphases is observed.

Statistical analysis: Descriptive statistic was used to analyze the mean, median, incidence and percentage .Kaplan-Meyer survival analysis was applied to determine the Progression-Free Survival (PFS) and Overall Survival (OS). PFS was calculated as the time elapsed between IM initiation and progression of the CML.OS was calculated the initiation of IM to death due to any cause.

\section{Results}

\section{Socio-demographic characteristics}

Thirty nine patients were admitted for CML in the hematology department of the Teaching Hospital. They were 25 male $(64.10 \%)$ and 14 were female $(35.90 \%)$ with a median age at the time of diagnosis of 36 years (range 16-69 years).

\section{Clinical and biological characteristics of patients with chronic myeloid leukemia}

The median duration of symptoms (time between beginning of the symptoms and admission at the department of hematology) was 11.4 months (range 0.5 and 50 months). The main complain at presentation was abdominal pain (51.28\%). The health performance status of the patient was assessed by the ECOG status (Eastern Cooperative Oncology Group). It was a grade 1 for 20 patients $(51.3 \%)$ at the time of presentation. Cells blood counts of patients showed high white cells count with an average of 168.5giga/l ( range 14.6 and 381) ), low hemoglobin rate at $8.4 \mathrm{~g} / \mathrm{dl}$ (range 3.1 and 12.8) and mean platelet rate at $415.4 \mathrm{~g} / 1(146$ and $998 \mathrm{G} / \mathrm{L})$. The prognostic score for survival of CML patients (Sokal score) was high for 18 patients $(46.2 \%)$. Thirty eight $(97.43 \%)$ on 39 patients presented at chronic phase. The median for follow-up time was 28 months (Table 1).

Table 1: Patient Charasteristics

\begin{tabular}{ll}
\hline Variable & $\mathbf{N}$ \\
\hline Time since diagnosis in month & $11.4(0.5-50)$ \\
Median (range) & \\
Main complaint; N (\%) & $20(51.28)$ \\
Abdominal pain & $7(17.95)$ \\
Fatigue & $2(5.13)$ \\
Headaches & $1(2.56)$ \\
weakness & $9(23.08)$ \\
No symptoms & \\
ECOG score: N (\%) & $19(48.72)$ \\
0 & $20(51.28)$ \\
1 & $16.4(0-28)$ \\
Median spleen size in cm (range) & $6.1(0-19)$ \\
Median hepatomegaly size in cm (range) & 8.4 \\
Median Hb rate in g/dl & $(3.1-12.8)$ \\
(range) & 168.5 \\
Median white cell count in giga/l & $(14.6-381)$ \\
(range) & 415.4 \\
Median platelet count in giga/l & $(146-998)$ \\
(range) & 28 \\
Median Follow up in month & $(5-68)$ \\
(range) & \\
SOKAL score & $7(17.9)$ \\
Low n (\%) & $14(35.9)$ \\
Intermediate n (\%) & $18(46.2)$ \\
High n (\%) & $38(97.43)$ \\
Chronic phase & $1(2.57)$ \\
Accelerated phase &
\end{tabular}




\section{Hematological response}

All patients achieved CHR in 3 months. Two patients $(5.13 \%)$ lost their CHR at 6 months while receiving Imatinib. At 1 year, 27 patients $(89.87 \%)$ continued to have a CHR (Table 2).

\section{Cytogenetic response}

At 6 months after the initiation of IM, 21 patients (88.18\%) achieved a Major Cytogenetic Response (MCYR). The response was complete (CCYR) in $20.51 \%$ and partial (PCYR) in $66.67 \%$ (Table 3)

\section{Outcomes}

After a median follow up of 28 months (range: 5 and 68 months), 6 patients (15.4\%) deceased, 1 was lost of follow $(2.6 \%)$ and $32(82.1 \%)$ were alive.

The projected 26 month Overall Survival rate (OS) was $86.6 \%$ (95\% CI: $34-71 \%$ ). The rate was $43 \%$ at 53 months (Figure 1).

8 of the 39 patients had progressed to the accelerated or blastic phase. The estimated rate of ProgressionFree Survival (PFS) was 91.8\% (95\%CI: 14-57\%) at 12 months and $43.6 \%$ at 36 months (Figure 2).

Table 2: Hematologic response

\begin{tabular}{lllll}
\hline $\begin{array}{l}\text { Hematologic } \\
\text { remission }\end{array}$ & 3 months & 6 months & 12 months & 24 months \\
\hline Total patient & 39 & 39 & 39 & 33 \\
Complete & $39(100 \%)$ & $37(94.87 \%)$ & $35(89.74 \%)$ & $27(81.82 \%)$ \\
Partial & 0 & $2(5.13 \%)$ & $4(10.26 \%)$ & $6(18.18 \%)$ \\
\hline
\end{tabular}

Table 3: Cytogenetic response

\begin{tabular}{ll}
\hline Variable & N $(\%)$ \\
\hline Complete n (\%) & $8(20.51)$ \\
Partial n (\%) & $26(66.67)$ \\
Minor n (\%) & $4(10.26)$ \\
No response n (\%) & $1(2.56)$ \\
Total & $39(100)$ \\
\hline
\end{tabular}

\section{Survival Function}

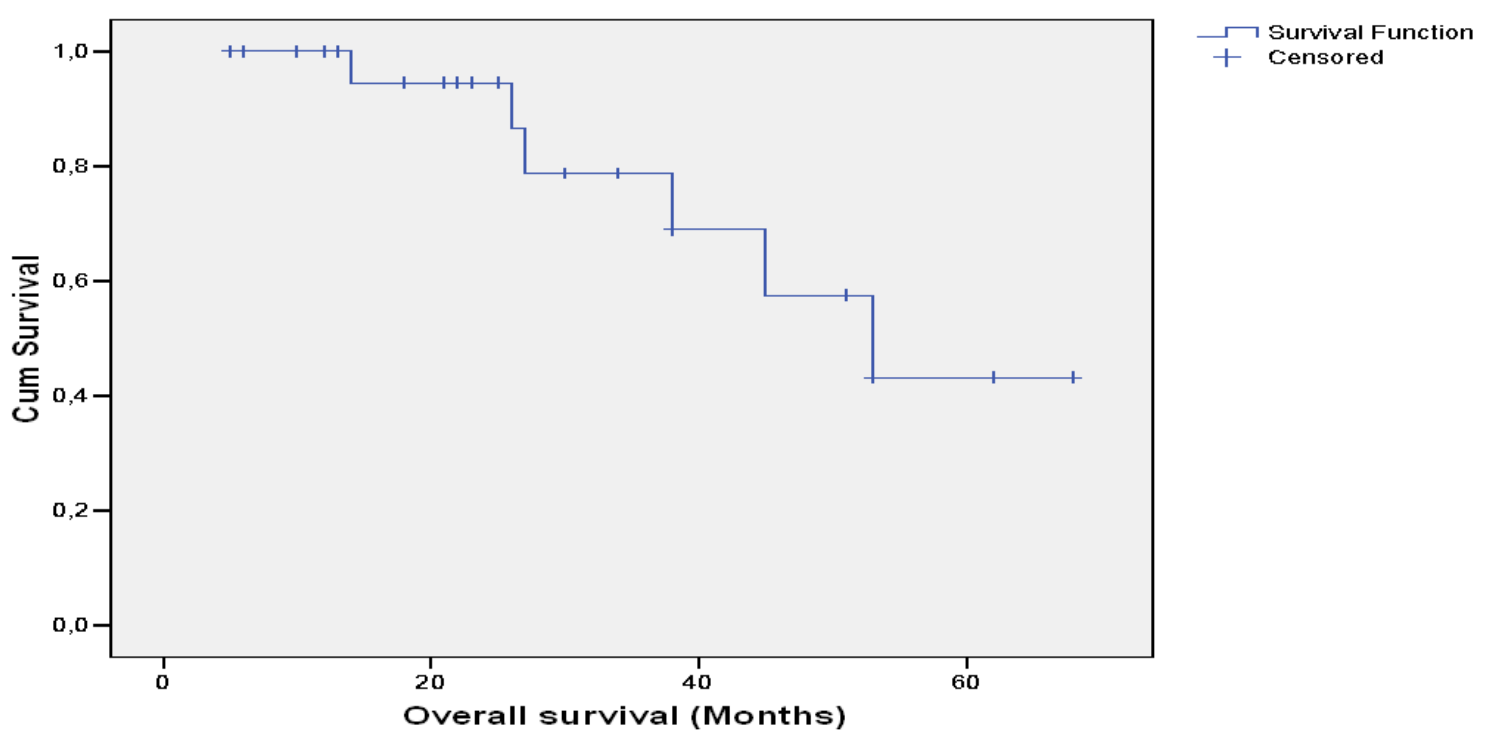

Figure 1: Landmark analysis of overall survival. 
Survival Function

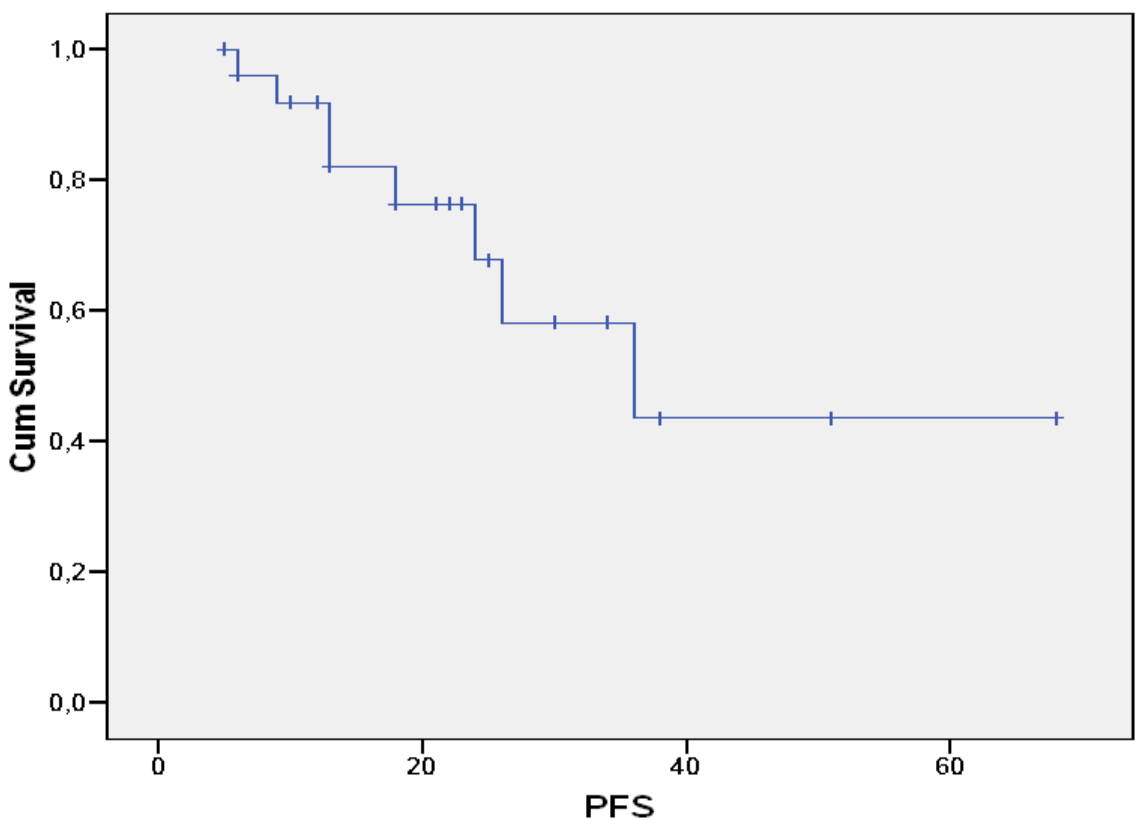

$\neg$ Survival Function

+ Censored

Figure 2: Landmark analysis of the Progression Free Survival

\section{Discussion}

This study aims to report clinical and biological feature of Congolese patients with chronic myeloid leukemia and complement previous studies in the African region (7-10). CML in low and middle resources countries shares some clinical and biological pattern that contrast with the one from western countries. CML affects younger population in low and middle resources countries (7-11). The median age at the presentation is a decade even two decades younger compared with the one in the West $(6,11$ 13). Indeed, the median age of onset goes from 37 to 42 years against the 65 years seen in the US and Europe (11-13). For Fleming and Menendez, this epidemiological distribution is a picture of the demographic distribution and is not relative to any inherent biological characteristics (14).

Because of the long delay diagnosis, 11.4 months our study, patients are symptomatic at the diagnostic and present in medical facilities with common symptoms as abdominal pain related to enlarge spleen (7-10). Splenomegaly is the most common finding followed by a hepatomegaly $(7-10,15)$. No organomegaly is noticed in only $5.4 \%$ (15). In comparison, the delay diagnosis is short in the West $(12,13,16)$. Consequently, $40 \%$ of patients are not symptomatic and diagnosis is made on the basis of abnormal blood count (16). Paradoxically, despite the long delay diagnosis reported, chronic phase is the most common stage of the disease noticed at the time of the presentation but prognosis is bad since $46.2 \%$ patients presented with high risk CML at the Sokal score in our series $(7-10,15)$.

The prognosis is better in Western countries since only $28.9 \%$ showed a high risk at the Sokal score $(12,13,16)$. The Sokal score evaluates the risk of death by including age, spleen size, platelet count and percentage of blasts circulating. The estimated survival is lower for high Sokal scores (17).

Leukocytosis is major and more pronounced with an average of $211.7 \mathrm{Giga} / \mathrm{l}$ in Ivory Coast (8). Anemia is moderate; the lowest hemoglobin has been reported by Segbena in Togo (9).

The hematological response is pretty good and complete hematological response is noticed in an average of 3 months (7-10). However cytogenetic response is not as impressive as reported in the West $(12,13,16)$. Data available in Africa region show poor performance of the imatinib (7-10) ). Only $20.51 \%$ of the patients in our trial achieved a CCYR at 6 months, $17 \%$ in Ivory Coast and $51 \%$ in Nigeria on patients that had previously interferon $(7,8)$. When we compare with the data in Asia, the cytogenetic response is broadly appreciated and ranges from $30 \%$ in India to $90 \%$ in Singapour (16).

The poorer performance is attributed on one hand to the inadequate cytogenetic monitoring that bias data (16). On the another hand, diagnosis at late chronic phase and poor initial prognosis are both statistically correlated to poor cytogenetic response $(8,17)$. Finally, additional chromosome abnormalities, $41 \%$ in the study of Koffi are associated with imatinib resistance (8). 
Nonetheless with the poor prognosis of CML in Africa, we found a comparable impressive projected OS rate of $94.4 \%$ and PFS at $91.8 \%$ at 12 months versus $96 \%$ and $91 \%$ in Nigeria (7) for the same duration of time. But, our values changed drastically at 53 months with an OS of $43 \%$ and a PFS of $43.6 \%$ at 36 months.

\section{Conclusion}

Chronic myeloid is the most prevalent chronic malignant blood disorder that affects young adults in Africa that present at late chronic phase with voluminous splenomegaly and major leukocytosis. Imatinib is an option treatment given at no cost to patients. Imatinib increased overall survival of patients, but the cytogenetic response remains lower compared to the West.

Conflict of Interest: The authors declare no potential conflicts of interest with respect to the research, authorship, and/or publication of this article.

Acknowledgements: The authors are grateful GIPAP for providing and facilitating the delivery of Imatinib in the Congo. We are also indebted to the Hematologic Laboratory in the Kremlin Bicêtre teaching hospital in Paris-France for the cytogenetic studies.

Author's Contributions: NLO drafted and the work. MF revised the manuscript, EDA approved the final version of the manuscript. All authors read and approved the final manuscript

Ethical issues: All Authors declare that Originality of research/article etc... and ethical approval of research, and responsibilities of research against local ethics commission are under the Authors responsibilities. The study was conducted due to defined rules by the Local Ethics Commission guidelines and audits.

\section{References}

1. Sawyers CL. Chronic myeloid leukemia. N. Engl J Med 1999; 340: 1330-40

2. Rowmey; "A new consistent chromosomal abnormality in chronic myelogenous leukemia identified by quinacrine fluorescence and Giemsa staining", Nature 1973; 5405 (243); 290-3.

3. Daley GQ, Van Etten RA, Baltimore D, "induction of chronic myelogenous leukemia in mice by the P210 (bcr/abl) gene of the Philadelphia chromosome". Science 1990; 4944 (247): 824-30
4. Redaelli A, Bell C, Casagrande J, Stephens J, Botteman M, Laskin $\mathrm{B}$ et al. Clinical and epidemiologic burden of chronic myelogenous leukemia. Expert Review of Anticancer Therapy 2014; 1(4): 85-96.

5. Nsonde Malanda J, Nkoua Mbon JB, Tozoulabambara A, Ibara G, Minga B, Nkoua Epala B et al. Douze années de fonctionnement du registre des cancers de Brazzaville. Bull Cancer. 2013; 2(100):135-39.

6. Druker B, Guilhot F, O’Brien GS, Gathmann I, Kantarjian $\mathrm{H}$, Gattermann N, et al. Five-year follow-up of imatinib therapy for newly diagnosed chronic myelogenous leukemia in chronic-phase shows sustained responses and high overall survival. N Engl J Med 2006; 355: 2408-17

7. Muheez AD, Faluyi JO, Oyekunle AA et al. The use of imatinib mesylate in Nigerians with chronic myeloid leukemia. Cellular Therapy and Transpantation 2008; 1 (2):.58-62.

8. Koffi KG, Nanho DC, N'dathz E et al. The effect of imatinib mesylate for newly diagnosed Philadelphia chromosome-positive, chronic-myeloid leukemia in SubSaharan African patients: the experience of Côte d'Ivoire. Advances in hematology 2010; 10 (268921): 1-6.

9. Segbena AY, Kueviakoe IMD, Agbetiafa K, Padaro E, ayibo Y, Dorkenoo A, Agbo YM. Leucémie myéloïde chronique et imatinib, experience du CHU Campus de Lomé au Togo. Médecine Santé et Tropicales 2012 ; 22(3) : 307-11.

10. Chemegni B, Tayou Tagne C, Nlend Nlend MC, Ngouadjeu E, Ngo Sack f, Kaptue Noche L et, al. La réponse hématologique chez les patientsatteints de leucémie myeloide chronique sous imatinib à Yaound2. Health Sci. Dis 2016 ; $17(1)$ : 1-6

11. Mendizabal AM, Naji Y, Levine PH. Geographic and income variation in age at diagnosis and incidence of chronic myeloid leukemia. International of Hematology 2016; 103(1): 70-8.

12. De Lavallade H, Khorashad JFA, Milokovic D et al Imatinib and newly diagnosed patients with chronic myeloid leukemia: incidence on sustained responses in an intention-to-treat analysis. Journal of Clinical Oncology 2008; 26 (20): 3358-63.

13. Kantarjian HM, Smith TL, McCredie KB et al. Chronic Myelogenous Leukemia: Mutlivariate Analysis of the Association of Patients Characteristics and Therapy with survival. Blood 1985; 66 (6): 1326-35.

14. Fleming AF and Menendez C. "blood", in principles of Medicine in Africa, Parry E, Godfrey R, Mabey D, and G. Gill, Eds., vol. 78,Cambridge University Press, Cambridge UK, 2004.

15. Prosanth $\mathrm{G}$ and Lalit K. Chronic myeloid leukemia in India. Journal of Global Oncology 2017; 3(1): 67-71.

16. Wing YA, Caguioa PB, Chuah C, chun Hsu S, Saengsunee J, Dong-Wook K et, al. Chronic myeloid leukemia in Asia. Int J Hematol 2009; 89: 14-23.

17. Sokal JE. Evaluation of survival data from chronic myelocytic leukemia. Am J Hematol 1976; 1 (4): 493-500. 